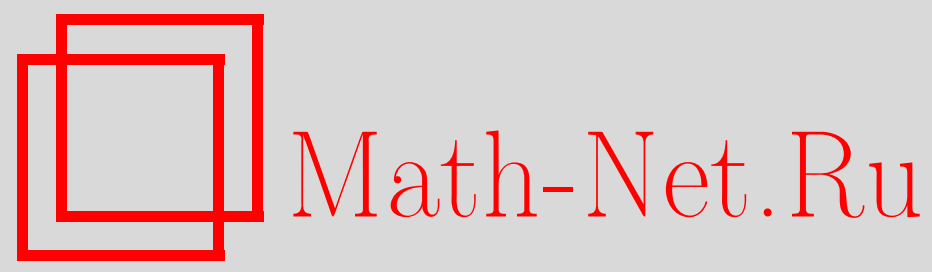

А. В. Борщев, Д. И. Молдаванский, Об изоморфизме некоторых групп с одним определяющим соотношением, $M a$ тем. заметки, 2006, том 79, выпуск 1, 34-44

DOI: https://doi.org/10.4213/mzm2672

Использование Общероссийского математического портала Math-Net.Ru подразумевает, что вы прочитали и согласны с пользовательским соглашением http://www . mathnet.ru/rus/agreement

Параметры загрузки:

IP : 3.85 .5 .30

26 апреля 2023 г., $13: 28: 13$

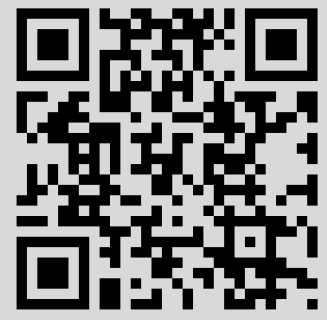




\title{
ОБ ИЗОМОРФИЗМЕ НЕКОТОРЫХ ГРУПП С ОДНИМ ОПРЕДЕЛЯЮЩИМ СООТНОШЕНИЕМ
}

\author{
А.В. Борщев, Д. И. Молдаванский
}

Рассматривается класс групп, каждая из которых задается одним определяющим соотношением, зависяшим от трех целочисленных параметров, и является HNN-pacшшрением некоторой группы Баумслага-Солитэра. Получены условия, необходимые и достаточные для того, чтобы группы этого класса были изоморфны, а также для того, чтобы каждая из двух неизоморфных групп являлась гомоморфньп образом другой. Следствием этого является отрицательный ответ на вопрос 3.33 из "Коуровской тетради".

Библиограффия: 7 названий.

Введение. В данной статье рассматриваются групшы вида

$$
G(l, m ; k)=\left\langle a, t ; t^{-1} a^{-k} t a^{l} t^{-1} a^{k} t=a^{m}\right\rangle,
$$

где $l, m$ и $k$ - произвольные целые числа, отличные от нуля. Систематическое изучение этих групп впервые предпринял А. М. Бруннер [1], отметивший, в частности, что каждая такая группа изоморфна некоторой группе $G(l, m ; k)$, числовые параметры $l, m$ и $k$, определяющие которую, удовлетворяют условиям $k>0$ и $|l| \geqslant m>0$ (и эти условия предполагаются вьполненными всюду ниже). Впрочем, на группы этого класса еще в 1969 году обратил внимание Г. Баумслаг [2], доказав, что все конечные гомоморфные образы групшы $G(2,1 ; 1)$ являются циклическими группами (и приведя тем самым еше один пример групш с одним определяюшим соотношением, не апроксимируемой конечными группами). Исследования А. М. Бруннера были продолжены затем в работе [3]: при $|l|>m$ найдены образуюшие и определяющие соотношения группы всех автоморфизмов групшы $G(l, m ; k)$ (в [1] это сделано в случаях, когда $m=1$ или $m$ не делит $l)$, доказано, что группа $G(l, m ; k)$ является финитно аппроксимируемой тогда и только тогда, когда $|l|=m$ (в [1] отмечена необходимость этого условия), и не является хопфовой тогда и только тогда, когда $|l|>m>1$, число $m$ является делителем чисел $l$ и $k$ и числа $m$ и $l / m$ взаимно просты (в [1] доказана достаточность этих условий). Наконец, в работе [4] показано, что группа $G(l, m ; k)$ аппроксимируема конечньми $p$-групшами тогда и только тогда, когда $|l|=m=p^{r}$ и $k=p^{s}$ для некоторых целых чисел $r \geqslant 0$ и $s \geqslant 0$, причем если $l=-m$, то $p=2$ и $s \leqslant r$.

Здесь мы рассматриваем вопрос об изоморфизме групп $G(l, m ; k)$. Нашей целью является доказательство следуюшего утверждения. 
Tеорема. 1) Если группь $G_{1}=G\left(l_{1}, m_{1} ; k_{1}\right)$ и $G_{2}=G\left(l_{2}, m_{2} ; k_{2}\right)$ гомоморфно отображаются друг на друга и выполнено хотя бы одно из неравенств $\left|l_{1}\right|>m_{1}$ $u\left|l_{2}\right|>m_{2}$, mo $_{1}=l_{2}$ u $m_{1}=m_{2}$.

2) Если $|l|>m$, то группь $G_{1}=G\left(l, m ; k_{1}\right)$ и $G_{2}=G\left(l, m ; k_{2}\right)$ изоморфны тогда и только тогда, когда выполнено одно из следующих условий:

(2.1) $k_{1}=k_{2}$

(2.2) $m>1$, числа $k_{1}$ и $k_{2}$ делятся на наибольший общий делитель чисел $l$ u $u k_{1} / k_{2}= \pm(l / m)^{p}$ для некоторого челого числа $p \neq 0$

(2.3) $m=1$ и частное $k_{1} / k_{2}$ является l-числом.

3) Группь $G_{1}=G\left(l, m ; k_{1}\right)$ и $G_{2}=G\left(l, m ; k_{2}\right)$ гомоморфно отображаются друг на друга и не изоморфны тогда и только тогда, когда $|l|>m>1$ и число $m$ является делителем каждого из чисел $l, k_{1}$ и $k_{2}$, причем число $s=l / m$ взаимно просто с $m$ и частное $k_{1} / k_{2}$ является $s$-числом, не совпадающим ни с какой степенью (с иелочисленным показателем) числа $\pm s$.

(Здесь, как обычно, утверждение о том, что целое число $m$ является $n$ - иислом, означает, что $m$ делится лишь на простые числа, делящие целое число $n$; рациональное число будем назьвать $n$-числом, если $n$-числами являются числитель и знаменатель представляющей его несократимой дроби.)

Попытка указать необходимые и достаточные условия существования изоморфизма между группами рассматриваемого класса была анонсирована в [5]. Однако формулировка приведенной там теоремы оказалась не вполне верной, и пункт 2) нашей теоремы можно рассматривать как ее уточнение в том случае, когда $\left|l_{1}\right|>m_{1}$ или $\left|l_{2}\right|>m_{2}$. Вопрос об условиях изоморфности групп $G_{1}$ и $G_{2}$ в оставшемся случае $\left|l_{1}\right|=m_{1}$ и $\left|l_{2}\right|=m_{2}$ остается открытым.

Заметим, впрочем, что если групшы $G_{1}$ и $G_{2}$ гомоморфно отображаются друг на друга и, скажем, $\left|l_{1}\right|=m_{1}$, то группа $G_{1}$, будучи финитно аппроксимируемой, является хопфовой, и потому группа $G_{2}$ должна быть изоморфной групше $G_{1}$. Следовательно, группа $G_{2}$ финитно аппроксимируема, и потому $\left|l_{2}\right|=m_{2}$. Если предположить, далее, что $l_{1}=-m_{1}$, то, переходя к фактор-группам по коммутантам, получаем $l_{1}=l_{2}$ и $m_{1}=m_{2}$. Таким образом, утверждение пункта 1$)$ нашей теоремы справедливо и в том случае, когда хотя бы для одного значения $i=1,2$ выполнено равенство $l_{i}=-m_{i}$. Одновременно мы видим, что выполнимость неравенства $|l|>m$ в утверждении пункта 3 ) нашей теоремы можно считать очевидной.

С другой стороны, приведенное в [5] следствие из упомянутой теоремы является справедливым. В нем указаны условия, достаточные для того, чтобы неизоморфные группы $G_{1}$ и $G_{2}$ гомоморфно отображались друг на друга (необходимые и достаточные условия даны в пункте 3 ) теоремы, сформулированной выше). Это позволяет дать отрицательный ответ на вопрос 3.33 из [6]: будут ли изоморфны две группы, каждая из которых задается одним определяюшим соотношением и является гомоморфным образом другой? Действительно, уже из условий, сформулированных в [5], следует, что контрпримером могут служить группы $G(18,2 ; 2)$ и $G(18,2 ; 6)$, а из пункта 3$)$ нашей теоремы следует, что этот пример является в определенном смысле минимальным.

Доказательству теоремы посвящен второй раздел статьи. В первом разделе приводится ряд необходимых нам утверждений, содержащихся в работах [1] и [3] или непо- 
средственно вытекающих из этих работ. Еще раз подчеркнем, что всюду ниже без дополнительных оговорок предполагается, что числовые параметры $l, m$ и $k$ (с одинаковыми индексами или без них) удовлетворяют неравенствам $|l|>m>0$ и $k>0$ (ограничение $|l|>m>0$ существенно, в частности, в ряде ссылок на результаты из [1]).

1. Предварительные замечания. Следуя [1], введем в представление $\langle a, t$; $\left.t^{-1} a^{-k} t a^{l} t^{-1} a^{k} t=a^{m}\right\rangle$ групшы $G(l, m ; k)$ новый образуюший $b$ вместе с определяющим соотношением $b=t^{-1} a^{k} t$. Полученное представление

$$
G(l, m ; k)=\left\langle a, b, t ; b^{-1} a^{l} b=a^{m}, t^{-1} a^{k} t=b\right\rangle
$$

делает очевидным тот факт, что группа $G(l, m ; k)$ является HNN-расширением с проходной буквой $t$ группы $H(l, m)=\left\langle a, b ; b^{-1} a^{l} b=a^{m}\right\rangle$, причем связанные подгрупшы $H^{-1}$ и $H^{1}$ являются бесконечными циклическими, порождаемьми элементами $a^{k}$ и $b$ соответственно (все необходимые определения и утверждения, относящиеся к конструкции HNN-расширения, можно найти в [7]). Каждая из групп $H(l, m)$ (назьваемых в литературе групшами Баумслага-Солитэра), в свою очередь, является HNN-расширением с проходной буквой $b$ бесконечной циклической группы, порождаемой элементом $a$. Поэтому нам придется говорить o $t$-длине и $t$-приведенной записи и о $b$-длине и $b$-приведенной записи элементов группы $G(l, m ; k)$.

Символом $L$ будем обозначать нормальное замыкание в групше $H(l, m)$ элемента $a$. Очевидно, что группа $H(l, m)$ является расщепляемым расширением подгруппы $L$ при помощи бесконечной циклической группы с образующим $b$ и что элемент $h \in H(l, m)$ принадлежит подгруппе $L$ в точности тогда, когда сумма показателей по $b$ в (произвольном) слове от образующих $a$ и $b$, представляющем элемент $h$, равна 0 . Договоримся также через $C(h)$ обозначать централизатор в групше $H(l, m)$ элемента $h$ этой групшы.

Рассматривая одновременно две группы вида $G(l, m ; k)$, будем записьвать $G_{1}=$ $G\left(l_{1}, m_{1} ; k_{1}\right)$ и $G_{2}=G\left(l_{2}, m_{2} ; k_{2}\right)$ и снабжать соответствуюшими индексами их образующие $a, b, t$ и подгруппы $H=H(l, m), H^{-1}, H^{1}$ и $L$.

Перечислим некоторые необходимые нам свойства групп $H(l, m)$ и $G(l, m ; k)$. Непосредственной индукцией по $b$-длине элемента $h$ доказывается следующее утверждение, являющееся некоторым уточнением леммы 2.4 из работы [1].

ПРЕДЛОЖЕНИЕ 1. Пусть әлемент $h \in H(l, m)$ записан в виде $h=b^{p} v$, әде $v \in L$, $p \in \mathbb{Z}$. Пусть $d=(l, m)$ - наибольший общий делитель чисел $l u m$ u $l=l_{1} d$, $m=m_{1} d$. Равенство $h^{-1} a^{r} h=a^{s}$ имеет место тогда и только тогда, когда $v \in C\left(a^{s}\right)$ и выполнено одно из следующих условий в зависимости от знака $p$ : $r=s$, если $p=0, r=l_{1}^{p} d x$ u $s=m_{1}^{p} d x$ для некоторого челого числа $x$, если $p>0$, $u r=m_{1}^{-p} d x$ us $=l_{1}^{-p} d x$ для некоторого челого числа $x$, если $p<0$.

ПРЕДЛОЖЕНИЕ $2[1$, лемма 2.3]. Пусть $h \in H(l, m)$. Если для некоторого целого числа $n \neq 0$ әлемент $h^{n}$ сопряжен $c$ әлементом из подгруппь, порожденной әлементом $a$, или из подгруппы, порожденной әлементом $b$, то $u$ әлемент $h$ сопряжен с некоторым әлементом из той же подгруппы.

ПРЕДЛОЖЕНИЕ 3. Пусть для некоторого әлемента $g \in G(l, m ; k)$ и некоторого иелого числа $n \neq 0$ имеет место включение $g^{-1} a^{n} g \in H(l, m)$. Тогда әлемент $g^{-1} a^{n} g$ принадлежит подгруппе $L$ в том и только том случае, когда әлемент $g$ принадлежит подгруппе $H(l, m)$. При этом, если $g \notin H(l, m)$, то әлементы $g$ 
$u g^{-1} a^{n} g$ имеют соответственно вид $g=b^{p} u t v u g^{-1} a^{n} g=v^{-1} b^{s} v$, әде $s \neq 0 u$ $p$ - некоторые челье числа, $u$ u $v$ - әлементы из подгруппь $L$, причем $u \in C\left(a^{k s}\right)$ $u b^{-p} a^{n} b^{p}=a^{k s}$.

В самом деле, если $g \in H(l, m)$, включение $g^{-1} a^{n} g \in L$ очевидно. Предположим, что $g \notin H(l, m)$. Так как по условию $g^{-1} a^{n} g \in H(l, m)$, из леммы 2.5 работы [1] следует, что тогда элемент $g$ имеет вид $g=w t v$, где $w \in H(l, m), v \in L$. Поскольку запись $v^{-1} t^{-1} w^{-1} a^{n} w t v$ элемента $g^{-1} a^{n} g$ не может быть $t$-приведенной (ввиду его принадлежности подгрупе $H(l, m))$, для подходящего целого числа $s \neq 0$ должно вьполняться равенство $w^{-1} a^{n} w=a^{k s}$. Поэтому $g^{-1} a^{n} g=v^{-1} b^{s} v$, и, так как $s \neq 0$, элемент $g^{-1} a^{n} g$ в этом случае действительно не входит в подгруппу $L$. Наконец, записывая элемент $w$ в виде $w=b^{p} u$, где $u \in L$, в силу предложения 1 имеем $u \in C\left(a^{k s}\right)$, и потому равенство $w^{-1} a^{n} w=a^{k s}$ принимает вид $b^{-p} a^{n} b^{p}=a^{k s}$.

Эндоморфизм $\varphi$ групшы $G(l, m ; k)$ назовем спеииальным, если $a \varphi=a^{r}$ и $b \varphi=b g$ для некоторого целого числа $r \neq 0$ и элемента $g \in L$. Число $r$ будем называть показателем эндоморфизма $\varphi$. Лемма 3.1 из работы [1] утверждает, в частности, что для любого эндоморфизма $\psi$ групшы $G(l, m ; k)$ с нециклическим образом существует внутренний автоморфизм $\tau$ этой группы такой, что произведение $\psi \tau$ является специальным эндоморфизмом. Кроме того, имеет место следующее утверждение, доказательство которого дословно повторяет соответствующую часть рассуждений, используемых в [1] при доказательстве этой леммы.

ПРЕДЛОЖЕНИЕ 4. Пусть $\varphi-$ специальный әндоморфизм әруппь $G(l, m ; k)$ с показателем $r$. Тогда для некоторого челого числа $p \geqslant 0$ выполнено равенство $r^{p}=l^{p}$ и әлемент $t \varphi$ имеет вид $b^{p} u t v$, дде $u, v \in L u u \in C\left(a^{k}\right)$. Kроме того, $b \varphi=v^{-1} b v$ ( $и$ потому әлемент $g$ из определения специального әндоморфизма совпадает с коммутатором $[b, v])$.

ПРЕДЛОЖЕНИЕ 5. 1) Каждый специальный әндоморфизм әруппь $G(l, m ; k)$ с показателем $r=1$ инвективен.

2) Если группа $G(l, m ; k)$ обладает специальным әндоморфизмом с показателем $r \neq \pm 1$, то число $m$ является делителем чисел $l u k$.

3) Если $m>1$ и специальный эндоморфизм с показателем $r \neq \pm 1$ сюрвективен, то он не является инвективным.

Все утверждения этого предложения можно найти в работе [3]; тем не менее, для удобства читателей ниже приводится их доказательство.

ДОКАЗАТЕЛЬСТВО ПРЕДЛОЖЕНИЯ 5. Пусть $\varphi$ - специальный эндоморфизм группы $G(l, m ; k)$ с показателем $r$. Таким образом, в силу определения $a \varphi=a^{r}$, а в силу предложения $4 b \varphi=v^{-1} b v$ и $t \varphi=b^{p} u t v$ для некоторого целого числа $p \geqslant 0$, удовлетворяющего равенству $r m^{p}=l^{p}$, и некоторых элементов $u, v \in L$, причем $u \in C\left(a^{k}\right)$.

Покажем, что если $r=1$, то эндоморфизм $\varphi$ инъективен. Очевидно, что подгруппа $H=H(l, m)$ является $\varphi$-допустимой, и мы покажем сначала, что при $r=1$ эндоморфизм $\varphi$ действует инъективно на этой подгрупше. Пусть элемент $h \in H$ c $b$-приведенной записью

$$
h=a^{s_{0}} b^{\varepsilon_{1}} a^{s_{1}} \ldots b^{\varepsilon_{n}} a^{s_{n}}
$$

принадлежит ядру эндоморфизма $\varphi$. Если $n \geqslant 1$, то, поскольку запись

$$
h \varphi=a^{s_{0}}\left(v^{-1} b v\right)^{\varepsilon_{1}} a^{s_{1}} \ldots\left(v^{-1} b v\right)^{\varepsilon_{n}} a^{s_{n}}
$$


элемента $h \varphi$ не может быть $b$-приведенной, для некоторого номера $i$ должно выполняться равенство $\varepsilon_{i}+\varepsilon_{i+1}=0$ и элемент $v a^{s_{i}} v^{-1}$ должен принадлежать подгрупше, порожденной элементом $a^{l}$, если $\varepsilon_{i}=-1$, или подгрупше, порожденной элементом $a^{m}$, если $\varepsilon_{i}=1$. Иначе говоря, для подходящего целого числа $x$ вьполнено равенство $v a^{s_{i}} v^{-1}=a^{l x}$, если $\varepsilon_{i}=-1$, или равенство $v a^{s_{i}} v^{-1}=a^{m x}$, если $\varepsilon_{i}=1$. Так как $v \in L$, из предложения 1 следует, что тогда при $\varepsilon_{i}=-1 s_{i}=l x$, а при $\varepsilon_{i}=1 s_{i}=m x$. Поскольку и то, и другое противоречит $b$-приведенности записи элемента $h$, имеем $n=0$. Но тогда $h \varphi=h$, и потому $h=1$.

Пусть теперь $g \in G(l, m ; k)$ - произвольный элемент ядра отображения $\varphi$ и пусть

$$
g=h_{0} t^{\varepsilon_{1}} h_{1} \cdots t^{\varepsilon_{n}} h_{n}
$$

- $t$-приведенная запись этого элемента. Так как при $r=1$ из равенства $r m^{p}=l^{p}$ (и неравенства $|l|>m>0$ ) следует, что $p=0$, имеем $t \varphi=u t v$, и потому образ элемента $g$ записьвается в виде

$$
g \varphi=\left(h_{0} \varphi\right)(u t v)^{\varepsilon_{1}}\left(h_{1} \varphi\right) \cdots(u t v)^{\varepsilon_{n}}\left(h_{n} \varphi\right) .
$$

Так как при $n \geqslant 1$ эта запись не может быть $t$-приведенной, то в этом случае для некоторого номера $i$ должно вьполняться равенство $\varepsilon_{i}+\varepsilon_{i+1}=0$, причем если $\varepsilon_{i}=-1$, то элемент $u^{-1}\left(h_{i} \varphi\right) u$ должен принадлежать подгруппе $H^{-1}$, а если $\varepsilon_{i}=1$, то элемент $v\left(h_{i} \varphi\right) v^{-1}$ должен принадлежать подгруппе $H^{1}$. Таким образом, при $\varepsilon_{i}=-1$ для некоторого целого числа $x$ вьполнено равенство $u^{-1}\left(h_{i} \varphi\right) u=a^{k x}$. Ввиду включения $u \in C\left(a^{k}\right)$ имеем тог да $h_{i} \varphi=a^{k x}=\left(a^{k x}\right) \varphi$, откуда в силу инъективности действия $\varphi$ на подгрупе $H$ получаем $h_{i}=a^{k x}$, что противоречит $t$-приведенности записи элемента $g$. Аналогично, при $\varepsilon_{i}=1$ должно выполняться равенство $v\left(h_{i} \varphi\right) v^{-1}=b^{x}$, откуда имеем $h_{i} \varphi=v^{-1} b^{x} v=\left(b^{x}\right) \varphi$, и потому $h_{i}=b^{x}$, что снова противоречит $t$-приведенности записи элемента $g$. Следовательно, длина $n t$-приведенной записи элемента $g$ оказьвается равной нулю, т.е. $g \in H$, и потому ввиду инъективности действия $\varphi$ на подгрупше $H$ имеем $g=1$. Утверждение 1 ) предложения 5 доказано.

Если предположить теперь, что $r \neq \pm 1$, то в равенстве $r m^{p}=l^{p}$ число $p$ будет положительным, и потому из этого равенства следует, что $m$ является делителем $l$. Равенство $(t \varphi)^{-1}(a \varphi)^{k}(t \varphi)=b \varphi$, переписанное в виде

$$
v^{-1} t^{-1} u^{-1} b^{-p} a^{k r} b^{p} u t v=v^{-1} b v
$$

после несложных преобразований принимает вид $a^{k r}=b^{p} a^{k} b^{-p}$. Так как $p>0$ и запись правой части не может быть $b$-приведенной, отсюда следует, что число $k$ должно делиться на $m$, и утверждение 2 ) также доказано.

Предположим, наконец, считая по-прежнему $r \neq \pm 1$, что $m>1$ и что эндоморфизм $\varphi$ сюръективен. Фиксируем произвольный элемент $g \in G(l, m ; k)$ такой, что $g \varphi=a$. Так как коммутатор $[g, a]$ при отображении $\varphi$ переходит в 1 , для доказательства утверждения 3) достаточно показать, что этот коммутатор является неединичным элементом группы $G(l, m ; k)$.

Пусть сначала элемент $g$ лежит в подгруппе $H$ и его $b$-приведенная запись имеет вид

$$
g=a^{s_{0}} b^{\varepsilon_{1}} a^{s_{1}} \cdots b^{\varepsilon_{n}} a^{s_{n}} .
$$


Так как $\left(a^{s_{0}}\right) \varphi=a^{r s_{0}} \neq a$, длина $n$ этой записи должна быть положительной. Но тогда запись

$$
a^{-s_{n}} b^{-\varepsilon_{n}} \cdots a^{-s_{1}} b^{-\varepsilon_{1}} a^{-1} b^{\varepsilon_{1}} a^{s_{1}} \cdots b^{\varepsilon_{n}} a^{s_{n}+1}
$$

коммутатора $[g, a]$ имеет положительную длину и является $b$-приведенной, поскольку $m>1$. Следовательно, в этом случае $[g, a] \neq 1$.

Осталось рассмотреть случай, когда $t$-длина элемента $g$ положительна. Пусть $t$-приведенная запись этого элемента имеет вид

$$
g=h_{0} t^{\varepsilon_{1}} h_{1} \cdots t^{\varepsilon_{n}} h_{n}
$$

где $n \geqslant 1$. Тогда коммутатор $[g, a]$ записывается в виде

$$
[g, a]=h_{n}^{-1} t^{-\varepsilon_{n}} \cdots h_{1}^{-1} t^{-\varepsilon_{1}} h_{0}^{-1} a^{-1} h_{0} t^{\varepsilon_{1}} h_{1} \cdots t^{\varepsilon_{n}} h_{n} a
$$

и мы покажем, что эта запись $t$-приведена.

Действительно, в противном случае к этой записи должны быть применимы $t$-редукции, а так как в группе $H$ элемент $h_{0}^{-1} a h_{0}$ не входит в подгруппу $H^{1}$, это возможно лишь в случае, когда $\varepsilon_{1}=1$ и $h_{0}^{-1} a h_{0}=a^{k x}$ для некоторого целого числа $x$. Из доказанного только что утверждения 2 ) следует, что сумма показателей по $a$ в любом слове, равном единище в группе $G(l, m ; k)$, должна делиться на $m$. Поэтому последнее равенство дает делимость на $m$ числа $k x-1$. Но это невозможно, так как $k$ делится на $m$ и $m>1$. Таким образом, и в этом случае коммутатор $[g, a]$ отличен от единицы, поскольку его $t$-длина положительна. Предложение 5 доказано.

2. Доказательство теоремы. Пусть групшы $G_{1}=G\left(l_{1}, m_{1}, k_{1}\right)$ и $G_{2}=G\left(l_{2}, m_{2}\right.$, $k_{2}$ ) являются гомоморфными образами друг друга и $\varphi-$ сюръективньй гомоморфизм группы $G_{1}$ на группу $G_{2}$. Тогда в групе $G_{2}$ должно вьполняться равенство

$$
\left(b_{1} \varphi\right)^{-1}\left(a_{1} \varphi\right)^{l_{1}}\left(b_{1} \varphi\right)=\left(a_{1} \varphi\right)^{m_{1}}
$$

и так как $\left|l_{1}\right|>m_{1}$, из леммы Коллинза [7, с. 254] следует, что элемент $a_{1} \varphi$ сопряжен с некоторым элементом из подгрупшы $H_{2}$. Поэтому, умножив отображение $\varphi$ на подходящий внутренний автоморфизм группы $G_{2}$, можно без потери общности считать, что $a_{1} \varphi \in H_{2}$. Покажем, что в действительности можно предполагать даже (снова домножая $\varphi$ на подходящий внутренний автоморфизм групшы $\left.G_{2}\right)$, что для некоторого целого числа $r_{1} \neq 0$ имеет место равенство

$$
a_{1} \varphi=a_{2}^{r_{1}}
$$

и что, кроме того, $b_{1} \varphi \in H_{2}$. В самом деле, если для отображения $\varphi$ включение $b_{1} \varphi \in H_{2}$ уже имеет место, то из равенства (1) и леммы Коллинза, примененной на этот раз к группе $H_{2}$, следует, что элемент $a_{1} \varphi$ сопряжен в $H_{2}$ с некоторой степенью элемента $a_{2}$. Если же $t_{2}$-длина элемента $b_{1} \varphi$ положительна, то из (1) и леммы Бриттона следует, что элемент $\left(a_{1} \varphi\right)^{l_{1}}$ сопряжен в групе $H_{2}$ с элементом одной из подгрупп $H_{2}^{-1}$ и $H_{2}^{1}$. В силу предложения 2 можно утверждать, что тогда и элемент $a_{1} \varphi$ сопряжен соответственно с некоторой степенью элемента $a_{2}$ или с элементом из подгруппы $H_{2}^{1}$. Так как подгруппы 
$H_{2}^{-1}$ и $H_{2}^{1}$ сопряжены в групше $G_{2}$, существование числа $r_{1}$, удовлетворяющего равенству (2), можно считать доказанным и в этом случае. При этом из сюръективности отображения $\varphi$ и нецикличности группы $G_{2}$ следует, что $r_{1} \neq 0$. Наконец, поскольку в силу (1) теперь имеет место включение $\left(b_{1} \varphi\right)^{-1} a_{2}^{r_{1} l_{1}}\left(b_{1} \varphi\right) \in L_{2}$, из предложения 3 получаем, что $b_{1} \varphi \in H_{2}$.

Так как образы элементов $a_{1}$ и $b_{1}$ лежат в подгруппе $H_{2}$, то ввиду сюръективности гомоморфизма $\varphi$ элемент $t_{1} \varphi$ в эту подгрупу не входит. Кроме того, выполненное в группе $G_{2}$ соотношение

$$
\left(t_{1} \varphi\right)^{-1}\left(a_{1} \varphi\right)^{k_{1}}\left(t_{1} \varphi\right)=b_{1} \varphi
$$

теперь принимает вид $\left(t_{1} \varphi\right)^{-1} a_{2}^{r_{1} k_{1}}\left(t_{1} \varphi\right)=b_{1} \varphi$, и поэтому из предложения 3 следует, что элементы $t_{1} \varphi$ и $\left(t_{1} \varphi\right)^{-1} a_{2}^{r_{1} k_{1}}\left(t_{1} \varphi\right)$ имеют вид $b_{2}^{p_{1}} u_{1} t_{2} v_{1}$ и $v_{1}^{-1} b_{2}^{s_{1}} v_{1}$ соответственно, где $s_{1} \neq 0$ и $p_{1}$ - некоторые целые числа, $u_{1}$ и $v_{1}$ - элементы из подгрупшы $L_{2}$, причем $u_{1} \in C\left(a_{2}^{k_{2} s_{1}}\right)$ и

$$
b_{2}^{-p_{1}} a_{2}^{r_{1} k_{1}} b_{2}^{p_{1}}=a_{2}^{k_{2} s_{1}}
$$

Следовательно, $b_{1} \varphi=v_{1}^{-1} b_{2}^{s_{1}} v_{1}$, и соотношение (1) принимает вид

$$
v_{1}^{-1} b_{2}^{-s_{1}} v_{1} a_{2}^{r_{1} l_{1}} v_{1}^{-1} b_{2}^{s_{1}} v_{1}=a_{2}^{r_{1} m_{1}}
$$

Теперь из предложения 1 (с учетом неравенств $\left|l_{i}\right|>m_{i}, i=1,2$ ) следует, что $s_{1}>0$ и для подходящего целого числа $x$ выполнены равенства

$$
r_{1} l_{1}=l_{21}^{s_{1}} d_{2} x, \quad r_{1} m_{1}=m_{21}^{s_{1}} d_{2} x
$$

где $d_{2}=\left(l_{2}, m_{2}\right), l_{2}=l_{21} d_{2}, m_{2}=m_{21} d_{2}$. Отсюда получаем

$$
\frac{l_{1}}{m_{1}}=\left(\frac{l_{2}}{m_{2}}\right)^{s_{1}} \text {. }
$$

Аналогичные рассуждения применимы и к произвольному сюръективному гомоморфизму $\psi$ групшы $G_{2}$ на группу $G_{1}$. В частности, можно считать, что для подходящих целых чисел $r_{2} \neq 0$ и $s_{2}>0$ и элемента $v_{2} \in L_{1}$ выполнены равенства $a_{2} \psi=a_{1}^{r_{2}}$, $b_{2} \psi=v_{2}^{-1} b_{1}^{s_{2}} v_{2}$ и что

$$
\frac{l_{2}}{m_{2}}=\left(\frac{l_{1}}{m_{1}}\right)^{s_{2}} \text {. }
$$

Из равенств (6) и (7) следует, что $l_{1} / m_{1}=\left(l_{1} / m_{1}\right)^{s_{1} s_{2}}$, и так как $\left|l_{1}\right|>m_{1}$, отсюда получаем $s_{1}=s_{2}=1$ и $l_{1} / m_{1}=l_{2} / m_{2}$.

Итогом этих рассуждений является

Лемма 1. Пусть группы $G_{1}=G\left(l_{1}, m_{1}, k_{1}\right)$ u $G_{2}=G\left(l_{2}, m_{2}, k_{2}\right)$ гомоморфно отображсаются друг на друга. Тогда $l_{1} / m_{1}=l_{2} / m_{2}$ и с точностью до мнохстелей, являющихся внутренними автоморфизмами соответствующих групп, произвольные сюрдективные гомоморфизмы $\varphi$ группы $G_{1}$ на группу $G_{2}$ и $\psi$ группы $G_{2}$ на группу $G_{1}$ таковьи, что $a_{1} \varphi=a_{2}^{r_{1}}, b_{1} \varphi=v_{1}^{-1} b_{2} v_{1} u a_{2} \psi=a_{1}^{r_{2}}, b_{2} \psi=v_{2}^{-1} b_{1} v_{2} \partial \Omega_{\text {s }}$ 
подходящих отличных от нуля иелых чисел $r_{1} u r_{2} u$ әлементов $v_{1} \in L_{2} u v_{2} \in L_{1}$. При этом для некоторых иелых чисел $p_{1}$ и $p_{2}$ имеют место соотношения

$$
\begin{array}{rll}
b_{2}^{-p_{1}} a_{2}^{r_{1} k_{1}} b_{2}^{p_{1}}=a_{2}^{k_{2}} & u & b_{1}^{-p_{2}} a_{1}^{r_{2} k_{2}} b_{1}^{p_{2}}=a_{1}^{k_{1}}, \\
v_{1}^{-1} b_{2}^{-1} v_{1} a_{2}^{r_{1} l_{1}} v_{1}^{-1} b_{2} v_{1}=a_{2}^{r_{1} m_{1}} & u & v_{2}^{-1} b_{1}^{-1} v_{2} a_{1}^{r_{2} l_{2}} v_{2}^{-1} b_{1} v_{2}=a_{1}^{r_{2} m_{2}}
\end{array}
$$

и для подходящих иелых чисел $x, y$ имеют место равенства

$$
r_{1} l_{1}=l_{2} x, \quad r_{1} m_{1}=m_{2} x \quad u \quad r_{2} l_{2}=l_{1} y, \quad r_{2} m_{2}=m_{1} y
$$

Кажсдый из әндоморфизмов $\varphi \psi$ группь $G_{1}$ и $\psi \varphi$ группы $G_{2}$ является специальным c показателем $r_{1} r_{2}$, и потому для некоторого иелого числа $p \geqslant 0$ выполнены равенства

$$
r_{1} r_{2} m_{1}^{p}=l_{1}^{p} \quad u \quad r_{1} r_{2} m_{2}^{p}=l_{2}^{p}
$$

В самом деле, поскольку равенства (8), (9) и (10) вытекают из равенств (3), (4) и (5) (и их аналогов для отображения $\psi$ ) соответственно, в доказательстве нуждается лишь последнее утверждение леммы 1. Покажем, например, что отображение $\varphi \psi$ является специальным эндоморфизмом группы $G_{1}$ с показателем $r_{1} r_{2}$. Действительно, равенство $a_{1}(\varphi \psi)=a_{1}^{r_{1} r_{2}}$ является очевидным, а так как $b_{1} \varphi=v_{1}^{-1} b_{2} v_{1}$ и $b_{2} \psi=v_{2}^{-1} b_{1} v_{2}$, где $v_{1} \in L_{2}$ и $v_{2} \in L_{1}$, имеем

$$
b_{1}(\varphi \psi)=\left(v_{1}^{-1} b_{2} v_{1}\right) \psi=\left(v_{2}\left(v_{1} \psi\right)\right)^{-1} b_{1}\left(v_{2}\left(v_{1} \psi\right)\right)=b_{1} g
$$

где $g=\left[b_{1}, v_{2}\left(v_{1} \psi\right)\right] \in L_{1}$. Существование числа $p \geqslant 0$, удовлетворяющего равенствам (11), следует теперь из предложения 4 и равенства $l_{1} / m_{1}=l_{2} / m_{2}$.

В следующих трех леммах предполагается, что $\varphi$ и $\psi$-сюръективные гомоморфизмы группы $G_{1}$ на группу $G_{2}$ и групшы $G_{2}$ на группу $G_{1}$ соответственно, имеющие вид, указанный в лемме 1 , а также используются связанные с ними обозначения и соотношения из формулировки этой леммы.

ЛЕмма 2. Пусть $\left|r_{1} r_{2}\right|=1$. Тогда $l_{1}=l_{2}, m_{1}=m_{2}$ и если $k_{1} \neq k_{2}$, то числа $k_{1}$ и $k_{2}$ делятся на наибольший общий делитель чисел $l_{1}$ и $m_{1}$ u $k_{1} / k_{2}= \pm\left(l_{1} / m_{1}\right)^{q}$ для некоторого челого числа $q \neq 0$.

В самом деле, поскольку тогда $r_{1}= \pm 1$, из равенств (10) следует, в частности, что $\pm m_{1}=m_{2} x$, и потому $m_{2}$ является делителем $m_{1}$. Аналогично, $m_{1}$ является делителем $m_{2}$, и потому $m_{1}=m_{2}$. Так как $l_{1} / m_{1}=l_{2} / m_{2}$, имеем $l_{1}=l_{2}$. Кроме того, первое из соотношений (8) дает равенство $b_{2}^{-p_{1}} a_{2}^{\varepsilon k_{1}} b_{2}^{p_{1}}=a_{2}^{k_{2}}$ (где $\varepsilon= \pm 1$ ), которое ввиду предложения 1 (и положительности чисел $k_{1}$ и $k_{2}$ ) имеет место тогда и только тогда, когда либо $k_{1}=k_{2}$, либо $p_{1} \neq 0$, числа $k_{1}$ и $k_{2}$ делятся на наибольший общий делитель чисел $l_{1}$ и $m_{1}$ и $k_{1} / k_{2}=\varepsilon\left(l_{1} / m_{1}\right)^{p_{1}}$.

ЛЕмма 3. Пусть $\left|r_{1} r_{2}\right|>1, m_{1}>1 u m_{2}>1$. Тогда $l_{1}=l_{2}, m_{1}=m_{2}$, число $m_{1}$ является делителем чисел $l_{1}, k_{1} u k_{2}$, число $s=l_{1} / m_{1}$ взаимно просто с $m_{1}$ и $k_{1} / k_{2}$ является s-числом. 
ДокАЗАТЕЛЬСТВо. Поскольку эндоморфизм $\varphi \psi$ групшы $G_{1}$ является специальньм с показателем $r_{1} r_{2}$, из пункта 2) предложения 5 следует, что число $m_{1}$ должно быть делителем чисел $l_{1}$ и $k_{1}$. Если $l_{1}=m_{1} s$, то в силу (11) имеем $r_{1} r_{2}=s^{p}$, причем $p>0$. Покажем, что числа $m_{1}$ и $s$ взаимно просты.

Так как эндоморфизм $\varphi \psi$ сюръективен, для некоторого элемента $w \in G_{1}$ мы должны иметь $a_{1}=w(\varphi \psi)$. Записьвая элемент $w$ в виде слова $w\left(a_{1}, b_{1}, t_{1}\right)$ от порождающих группы $G_{1}$, получаем равенство $a_{1}=v\left(a_{1}, b_{1}, t_{1}\right)$, где слово

$$
v\left(a_{1}, b_{1}, t_{1}\right)=w\left(a_{1}^{s^{p}}, b_{1}(\varphi \psi), t_{1}(\varphi \psi)\right)
$$

получено заменой в слове $w\left(a_{1}, b_{1}, t_{1}\right)$ каждого образующего некоторьм словом, равным его $\varphi \psi$-образу.

Подсчитаем сумму показателей по $a_{1}$ в слове $v\left(a_{1}, b_{1}, t_{1}\right)$. Отметим, прежде всего, что в силу предложения 4 можно считать, что в слове, определяющем элемент $b_{1}(\varphi \psi)$, сумма показателей по $a_{1}$ равна 0 . Далее, из вида элемента $t_{1}(\varphi \psi)$, указанного в предложении 4 , следует, что сумма показателей по $t_{1}$ в слове $v\left(a_{1}, b_{1}, t_{1}\right)$ совпадает с суммой показателей по $t_{1}$ в слове $w\left(a_{1}, b_{1}, t_{1}\right)$. С другой стороны, сумма показателей по $t_{1}$ в произвольном слове, равном единице в групе $G_{1}$, должна быть равна 0 . Из равенства $a_{1}=v\left(a_{1}, b_{1}, t_{1}\right)$ поэтому следует, что сумма показателей по $t_{1}$ в слове $v\left(a_{1}, b_{1}, t_{1}\right), \mathrm{a}$ потому и в слове $w\left(a_{1}, b_{1}, t_{1}\right)$, равна 0 . Таким образом, сумма показателей по $a_{1}$ в слове $v\left(a_{1}, b_{1}, t_{1}\right)$ равна $n s^{p}$, где $n$ - сумма показателей по $a_{1}$ в слове $w\left(a_{1}, b_{1}, t_{1}\right)$.

Так как число $m_{1}$ является делителем чисел $l_{1}$ и $k_{1}$, сумма показателей по $a_{1}$ в определяющих словах групшы $G_{1}$, а потому и в произвольном слове, равном единице в этой группе, кратна числу $m_{1}$. Поэтому из равенства $a_{1}=v\left(a_{1}, b_{1}, t_{1}\right)$ следует сравнение $n s^{p} \equiv 1\left(\bmod m_{1}\right)$, из которого, в свою очередь, следует взаимная простота чисел $m_{1}$ и $s$.

Те же рассуждения применительно к эндоморфизму $\psi \varphi$ группы $G_{2}$ (с учетом равенства $\left.l_{1} / m_{1}=l_{2} / m_{2}\right)$ дают равенство $l_{2}=m_{2} s$ и взаимную простоту чисел $m_{2}$ и $s$.

Второе из равенств (10) говорит о том, что число $m_{2}$ является делителем числа $r_{1} m_{1}$. Но так как $r_{1} r_{2}=s^{p}$, то $r_{1}$ является $s$-числом и потому взаимно просто с $m_{2}$. Следовательно, $m_{2}$ является делителем числа $m_{1}$. Аналогично, $m_{1}$ является делителем $m_{2}$, откуда и получаем $m_{1}=m_{2}$ и $l_{1}=l_{2}$.

Наконец, так как $l_{1}=m_{1} s$, из первого из соотношений (8) и предложения 1 следует, что $r_{1} k_{1}=s^{p_{1}} k_{2}$. Поэтому $k_{1} / k_{2}$ является $s$-числом, и лемма 3 доказана.

ЛЕмма 4. Если одно из чисел $m_{1}$ и $m_{2}$ равно 1, то и другое равно 1. Если $m_{1}=m_{2}=1$, mо $l_{1}=l_{2}$ и $k_{1} / k_{2}$ является $l_{1}$-числом.

ДокАЗАТЕЛЬСТво. Предположим, напротив, что $m_{1}>1$ и $m_{2}=1$. Из леммы 2 следует, что тогда $\left|r_{1} r_{2}\right|>1$, и потому ввиду предложения 5 эндоморфизм $\varphi \psi$ группы $G_{1}$ не является инъективньп.

С другой стороны, из (11) следует, что $r_{1} r_{2}=l_{2}^{p}$. Поэтому, произведение эндоморфизма $\psi \varphi$ на внутренний автоморфизм группы $G_{2}$, производимый элементом $b_{2}^{p}$, оставляет элемент $a_{2}$ неподвижным, т.е. является специальным эндоморфизмом с показателем 1. Из предложения 5 теперь следует, что эндоморфизм $\psi \varphi$ инъективен, откуда ввиду сюръективности $\psi$ следует инъективность отображений $\varphi$ и $\psi$, а потому и отображения $\varphi \psi,-$ противоречие. Первое утверждение леммы доказано. 
Если теперь $m_{1}=m_{2}=1$, то очевидно, что $l_{1}=l_{2}$. Так как в этом случае первое из соотношений (8) равносильно равенству $r_{1} k_{1}=l_{1}^{p_{1}} k_{2}$ и так как в силу (11) $r_{1}$ является $l_{1}$-числом, то $k_{1} / k_{2}$ является $l_{1}$-числом, и лемма 4 доказана.

Перейдем теперь непосредственно к доказательству утверждений теоремы.

Пусть группы $G_{1}=G\left(l_{1}, m_{1}, k_{1}\right)$ и $G_{2}=G\left(l_{2}, m_{2}, k_{2}\right)$ гомоморфно отображаются друг на друга. Тогда по лемме 1 имеет место равенство $l_{1} / m_{1}=l_{2} / m_{2}$, и если $m_{1}=m_{2}=1$, то и $l_{1}=l_{2}$. В оставшемся (ввиду леммы 4) случае, когда $m_{1}>1$ и $m_{2}>1$, утверждение пункта 1$)$ следует из лемм 2 и 3.

Для доказательства утверждения пункта 2) предположим сначала, что групшы $G_{1}=$ $G\left(l, m, k_{1}\right)$ и $G_{2}=G\left(l, m, k_{2}\right)$ изоморфны, и пусть $\varphi$ и $\psi$ - изоморфные отображения соответственно групшы $G_{1}$ на группу $G_{2}$ и группы $G_{2}$ на группу $G_{1}$. Без потери общности можно предполагать, что эти отображения имеют вид, указанный в лемме 1 . Пусть $k_{1} \neq k_{2}$. Так как при $m>1$ ввиду биективности отображения $\varphi \psi$ из предложения 5 следует равенство $\left|r_{1} r_{2}\right|=1$, то справедливость утверждения (2.2) следует из леммы 2 . Утверждение (2.3) является следствием леммы 4.

Обратно, предположим сначала, что вьполнено условие $(2.2)$, т.е. числа $k_{1}$ и $k_{2}$ делятся на наибольший общий делитель $d$ чисел $l$ и $m$ и для подходящих $\varepsilon= \pm 1$ и целого числа $p \neq 0$ выполнено равенство $k_{1} / k_{2}=\varepsilon(l / m)^{p}$. Легко видеть, что тогда для некоторого целого числа $x \quad \varepsilon k_{1}=l_{1}^{p} d x$ и $k_{2}=m_{1}^{p} d x$, если $p>0$, и $\varepsilon k_{1}=m_{1}^{-p} d x$ и $k_{2}=l_{1}^{-p} d x$, если $p<0$ (где $l_{1}=l / d$ и $m_{1}=m / d$ ). Непосредственно проверяется (см., впрочем, предложение 1 ), что в любом случае в групшах $G_{2}$ и $G_{1}$ вьполнены равенства $b_{2}^{-p} a_{2}^{\varepsilon k_{1}} b_{2}^{p}=a_{2}^{k_{2}}$ и $b_{1}^{p} a_{1}^{\varepsilon k_{2}} b_{1}^{-p}=a_{1}^{k_{1}}$ соответственно. Поэтому отображения

$$
a_{1} \mapsto a_{2}^{\varepsilon}, \quad b_{1} \mapsto b_{2}, \quad t_{1} \mapsto b_{2}^{p} t_{2} \quad \text { и } \quad a_{2} \mapsto a_{1}^{\varepsilon}, \quad b_{2} \mapsto b_{1}, \quad t_{2} \mapsto b_{1}^{-p_{t}} t_{1}
$$

определяют взаимно обратные изоморфизмы группы $G_{1}$ на групшу $G_{2}$ и групшы $G_{2}$ на группу $G_{1}$ соответственно.

Если теперь имеет место условие (2.3), то для подходяших целых $l$-чисел $x$ и $y$ выполнено равенство $x k_{1}=y k_{2}$. Выберем целое число $p>0$ так, чтобы для некоторого целого $z$ вьполнялось равенство $l^{p}=y z$. Тогда при $r=x z$ имеем $r k_{1}=l^{p} k_{2}$, и снова непосредственная проверка показьвает, что в группе $G_{2}$ вьполнено равенство $b_{2}^{-p} a_{2}^{r k_{1}} b_{2}^{p}=a_{2}^{k_{2}}$. Поэтому отображение $a_{1} \mapsto a_{2}^{r}, b_{1} \mapsto b_{2}, t_{1} \mapsto b_{2}^{p} t_{2}$ определяет гомоморфизм групшы $G_{1}$ в групшу $G_{2}$. Легко видеть при этом, что поскольку $r$ является $l$-числом, этот гомоморфизм сюръективен. Аналогично устанавливается существование сюръективного гомоморфизма групшы $G_{2}$ в групп $G_{1}$. Но так как $m=1$, группы $G_{1}$ и $G_{2}$ хопфовы (в силу сформулированного во введении критерия из работы [3]), и потому они изоморфны. Утверждение пункта 2) теоремы доказано полностью.

Для доказательства утверждения пункта 3$)$ предположим сначала, что групшы $G_{1}=$ $G\left(l, m, k_{1}\right)$ и $G_{2}=G\left(l, m, k_{2}\right)$ неизоморфны и что $\varphi$ и $\psi$ - сюръективные гомоморфные отображения, указанного в лемме 1 вида, групшы $G_{1}$ на группу $G_{2}$ и группы $G_{2}$ на группу $G_{1}$ соответственно. Из лемм 2 и 4 и уже доказанных утверждений пункта 2) получаем, что $\left|r_{1} r_{2}\right|>1$ и $m>1$. Поэтому необходимость условий утверждения пункта 3 ) следует из леммы 3 и того, что если бы при этом число $k_{1} / k_{2}$ являлось степенью числа $\pm s$, то в силу утверждения (2.2) группы $G_{1}$ и $G_{2}$ оказались бы изоморфньми.

Обратно, покажем, что если $l=m s$, числа $m$ и $s$ взаимно просты, $m$ является делителем каждого из чисел $k_{1}$ и $k_{2}$ и $x k_{1}=y k_{2}$ для некоторых целых $s$-чисел $x$ и $y$, то группы 
$G_{1}$ и $G_{2}$ гомоморфно отображаются друг на друга. Пусть целое число $p>0$ таково, что $s^{p}=y z$ для некоторого целого $z$. Так как тогда $k_{1} r=s^{p} k_{2}$, где $r=x z$, и числа $k_{1}$ и $k_{2}$ делятся на $m$, в групе $G_{2}$ вьполнено соотношение $b_{2}^{-p} a_{2}^{k_{1} r} b_{2}^{p}=a_{2}^{k_{2}}$. Следовательно, отображение

$$
a_{1} \mapsto a_{2}^{r}, \quad b_{1} \mapsto b_{2}, \quad t_{1} \mapsto b_{2}^{p} t_{2}
$$

определяет гомоморфизм групшы $G_{1}$ в группу $G_{2}$. Для доказательства сюръективности этого гомоморфизма достаточно понять, что для любого $s$-числа $r$ элементы $a_{2}^{r}$ и $b_{2}$ порождают групу $H_{2}$. Пусть, в самом деле, $n$ - наименьшее положительное $s$-число такое, что подгрупша группы $H_{2}$, порожденная этими элементами, содержит $a^{n}$. Предположив, что $n>1$, обозначим через $q$ простой делитель числа $n$. Тогда, записьвая $n=n_{1} q$ и $s=s_{1} q$, видим, что этой подгруппе принадлежит элемент $b_{2}^{-1} a_{2}^{n m s_{1}} b_{2}=$ $b_{2}^{-1} a_{2}^{n_{1} m s} b_{2}=a_{2}^{n_{1} m}$, а потому и элемент $a_{2}^{n_{1}}$. Таким образом, $n=1$, и сюръективность гомоморфизма доказана. Аналогично доказывается существование сюръективного гомоморфизма группы $G_{2}$ в группу $G_{1}$.

Остается заметить, что если, к тому же, $m>1$ и число $k_{1} / k_{2}$ не является степенью числа $\pm s$, то группы $G_{1}$ и $G_{2}$ в силу условия $(2.2)$ не изоморфны, и теорема полностью доказана.

\section{СПИСОК ЦИТИРОВАННОЙ ЛИТЕРАТУРЫ}

[1] Brunner A. M. On a class of one-relator groups // Canad. J. Math. 1980. V. 32. № 2. P. 414-420.

[2] Baumslag G. A noncyclic one-relator group all of whose finite quotients are cyclic // J. Austral. Math. Soc. 1969. V. 10. №3-4. P. 497-498.

[3] Кавуцкий М. А., Молдаванский Д. И. Об одном классе групп с одним определяющим соотношением // Алгебраические и дискретные системы. Межвузовский сб. научн. тр. Ивановского гос. ун-та. Иваново, 1988. С. 35-48.

[4] Молдаванский Д. И. Аппроксимируемость конечньпи $p$-группами HNN-расширений // Вестн. Ивановского гос. ун-та. 2000. № 3. С. 129-140.

[5] Борщев А. В. О проблеме изоморфизма для одного класса групп с одним определяющим соотношением // Международная алгебраическая конференция, посвященная памяти Д. К. Фаддеева. Тезисы докладов. С.-Петербург, 1997. С. 170-171.

[6] Нерешенные вопросы теории групп. Коуровская тетрадь. Изд. 15-е. Новосибирск, 2002.

[7] Линдон Р., Шупп П. Комбинаторная теория групп. М.: Мир, 1980.

Ивановский государственный университет

Поступило

E-mail : moldav@ivanovo.ac.ru

27.10 .2003

Исправленный вариант

10.05 .2005 